\title{
Nosocomial infection with Clostridium difficile investigated by pyrolysis mass spectrometry
}

\author{
T. D. I. CARTMILL, K. ORR* R. FREEMAN $\dagger$, P. R. SISSON $\ddagger$ and N. F. LIGHTFOOT
}

Department of Microbiology, North Manchester General Hospital, Manchester, * Royal Victoria Infirmary, † Medical School and $\ddagger$ Regional Public Health Laboratory, Newcastle upon Tyne

\begin{abstract}
Summary. Fifty-eight isolates of Clostridium difficile from two distinct outbreaks were examined for inter-strain similarity by pyrolysis mass spectrometry (PMS). The first outbreak began on a geriatric acute unit and spread to a long stay geriatric facility. PMS analysis showed that most isolates from both sites were indistinguishable. Isolates obtained in the preceding year from the long stay facility were found to be closely similar to these outbreak isolates. In the second, smaller outbreak on a female medical ward in another general hospital, PMS again showed that a single strain was probably responsible. Representative isolates from these two different outbreaks were shown to be distinct. The ability to compare rapidly large numbers of isolates of $C$. difficile makes PMS attractive for initial screening in suspected outbreaks, providing important information for outbreak management and allowing conventional typing methods to be concentrated on relevant isolates.
\end{abstract}

\section{Introduction}

Clostridium difficile is the primary cause of pseudomembranous colitis (PMC) and antibiotic-associated colitis (AAC). There is good epidemiological evidence of nosocomial outbreaks of infection with this organism that may extend into after-care facilities when infected or colonised patients are transferred. ${ }^{1}$ Therefore, the ability to identify and trace individual strains of $C$. difficile is an important goal. Various methods for typing $C$. difficile have been evaluated, including SDS-PAGE autoradiography of ${ }^{35} \mathrm{~S}$-methioninelabelled proteins, ${ }^{2}$ immunoblotting, ${ }^{3}$ serotyping, ${ }^{4}$ phage typing ${ }^{5}$ and restriction endonuclease DNA analysis (REA). ${ }^{6,7}$

Pyrolysis mass spectrometry (PMS) has been used recently for rapid inter-strain comparison in a range of outbreaks with many different bacterial species. We have investigated the potential use of PMS in outbreaks of nosocomial infection with $C$. difficile, using isolates collected from two epidemiologically suspected outbreaks occurring in different geographical areas and in different years.

\section{Materials and methods}

\section{C. difficile strains}

Isolates of $C$. difficile from a large suspected outbreak, involving more than one hospital site in the north west of England in 1991 (outbreak 1) and from a smaller ward-based incident in the north east of England in 1990 (outbreak 2) were investigated.

Outbreak 1. In Feb. 1991, an increased incidence of diarrhoea was noted on the geriatric acute unit of a large general hospital (GAU) in the north west of England. $C$. difficile was isolated from nine affected patients between $2 \mathrm{Feb}$. and 18 March 1991 and $C$. difficile toxin was detected in each case. No other intestinal pathogens were found and it was concluded that the diarrhoea was $C$. difficile-associated. One further isolate was made from a tenth patient on 3 May. C. difficile was re-isolated on 16 March from a patient with continuing symptoms who had been found to be positive first on 13 Feb. All 11 GAU isolates were retained in cooked meat broth pending further examination as Batch 1.

Patients from GAU were regularly transferred to any of four wards in a long stay geriatric hospital (LSG) for continuing care. An increase in incidence of C. difficile-associated diarrhoea was noted in this second hospital, beginning shortly after the presumed outbreak in the GAU; 24 isolates of $C$. difficile were obtained from 16 LSG patients between $15 \mathrm{Feb}$. and 8 May. Repeat isolates from some patients were obtained because of continuing symptoms or clinical relapse. In all but two instances, $C$. difficile cytotoxin was detected in the stool samples from which the organism was isolated. These LSG isolates formed Batch 2.

A further 10 isolates of $C$. difficile from the laboratory serving the general and long stay hospitals 

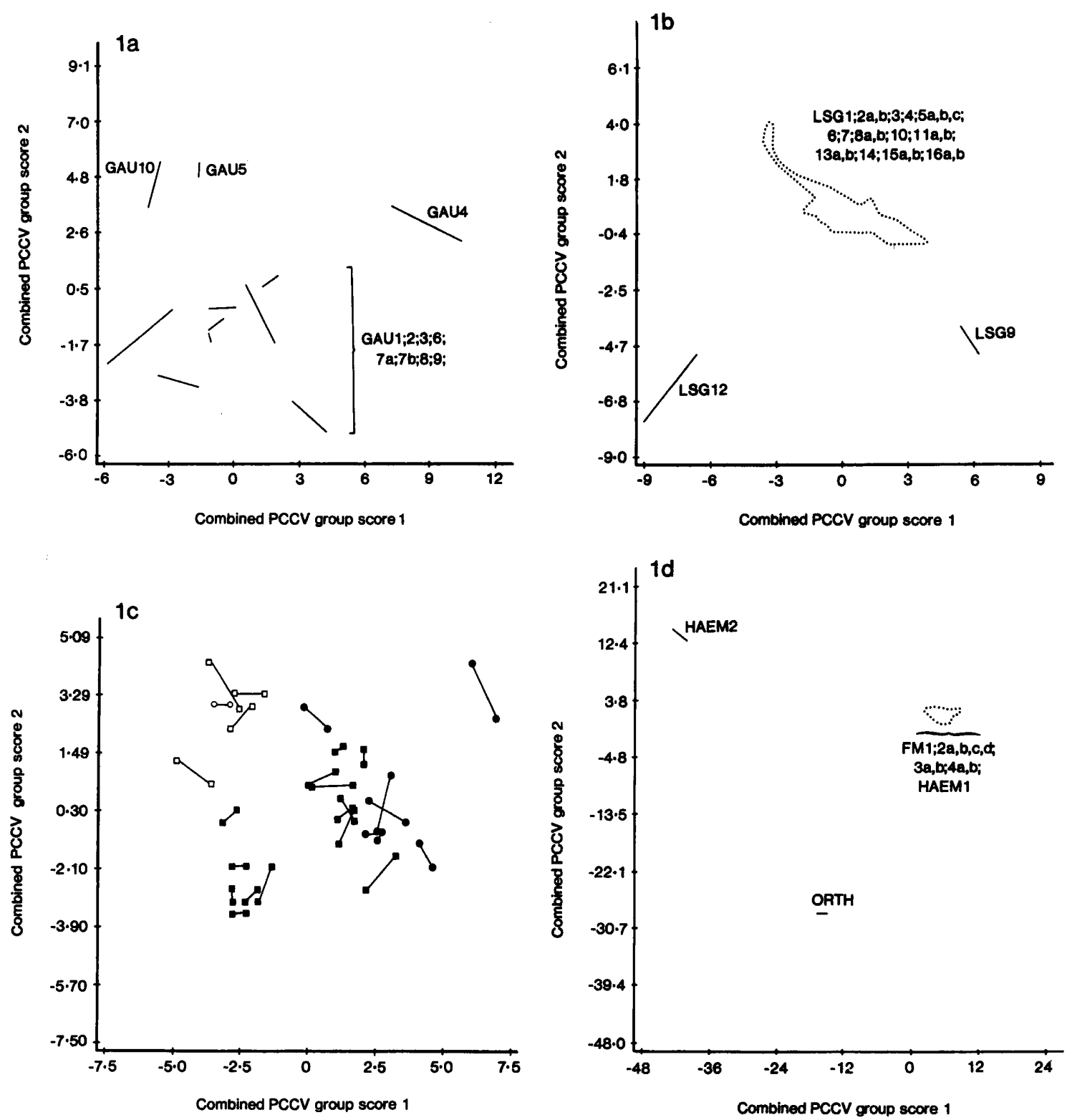

Fig. 1. Ordination diagrams of spectral data from PMS analyses. (a) Eleven isolates (GAU 1-11) of $C$. difficile from 10 patients involved in a suspected outbreak in a geratric acute unit (GAU); repeat isolates from a single patient are labelled GAU 7a and b.(b) Twenty-four isolates (LSG 1-16) of $C$. difficile from 16 patients in a long stay geriatric hospital (LSG); for patients LSG 2, 8, 11, 13, 15 and 16, two isolates were included, labelled in each case a and b; for patient LSG 5, three isolates were included, labelled LSG 5a, b and c. (c) Twenty-six isolates of C. difficile representing clusters of isolates identified in earlier analyses: GAU (O), seven isolates; LSG 1991 ( $\square$ ), 14 isolates; LSG 1990 ( $\square$ ), four isolates; and GM 1990 (O), one isolate. (d) Twelve isolates of $C$. difficile, comprising nine isolates from four patients (FM 1, 2, 3 and 4 ) in an outbreak of $C$. difficile-associated colitis on a female medical ward, two isolates from paediatric haematology patients (HAEM 1 and 2 ) and one isolate from an orthopaedic patient (ORTH); multiple isolates from patients FM 2, 3 and 4 are labelled FM $2 a, b$, etc. In all the ordination diagrams the means of the triplicate analyses for duplicate subcultures are joined.

were available. These included four sporadic isolates obtained from four different LSG patients between April and Dec. 1990 (LSG 90/1, 90/2, 90/3 and 90/4), two epidemiologically unrelated isolates from surgical patients in an adjoining health district, two isolates from general medical patients in wards other than the GAU in the general hospital in 1990 (GM 90/1 and $90 / 2$ ), one isolate from a general medical patient in 1991 (GM 91) and one isolate from the GAU obtained in Aug. 1990 (GAU 90). These isolates comprised Batch 3.

Outbreak 2. On 21 Feb. 1990 a patient developed
PMC (confirmed by sigmoidoscopy and biopsy) on an adult female medical ward in a large acute general hospital in the north east of England. C. difficile was isolated from the stool. Between 3 and 9 March 1990 a further three patients also developed histologically confirmed PMC and $C$. difficile was isolated in each instance; in all three cases the organism was re-isolated from stool samples obtained over the ensuing few days. Ten isolates from the four patients were preserved in cooked meat broth. These isolates were designated FM 1-4, with repeated isolates designated, e.g., FM 2a, 2b, etc. Concurrently, the same laboratory had isolated $C$. difficile from three epidemiologically 
unrelated patients with colitic symptoms-two paediatric haematology patients (isolates HAEM 1 and 2) and one adult orthopaedic patient (isolate ORTH). These three isolates were preserved similarly and then examined. These 13 isolates formed Batch 4.

Isolates from both outbreaks had been preserved in cooked meat broth at the end of initial routine diagnostic procedures and purity checks. In most instances isolates had not been subcultured until the time of this study.

\section{Pyrolysis mass spectrometry}

Each batch of isolates was cultured anaerobically overnight on Columbia Blood Agar (Lab M) at $37^{\circ} \mathrm{C}$. Smears were made on to pyrolysis foils (Horizon Instruments Ltd, Heathfield, Sussex) which were inserted into pyrolysis tubes (Horizon Instruments) and heated at $80^{\circ} \mathrm{C}$ for $5 \mathrm{~min}$. Triplicate smears were made from each of two subcultures of each isolate. Pyrolysis was then performed on a Horizon Instruments PYMS 200X pyrolysis mass spectrometer at a Curie point of $530^{\circ} \mathrm{C}$, as described previously. ${ }^{8}$ Integrated ion counts at unit mass intervals from 11 to 200 together with the pyrolysis sequence number and total ion count for each sample were recorded. Initially, each batch was examined separately but always within a single machine run. Following the initial analyses, further batches were assembled (see below) and examined similarly.

\section{Data analysis}

Spectra were normalised by an iterative technique to eliminate variation due to differences in the amount of sample pyrolysed. ${ }^{8}$ After normalisation replicate spectra of the same subculture were labelled as distinct groups and analysed for between-strain to withinstrain variation. The 30 masses showing the greatest discrimination between strains were then subjected to principal component and then canonical variate analyses, differences being expressed as a series of Mahalanobis distances. These data were used to construct ordination diagrams that were inspected for evidence of clustering.

Edited data sets containing only data from those isolates thought to be part of a cluster were reanalysed. The end point of these analyses was a number of isolates in which the differences found between two subcultures of the same organism were at least as great as any differences between subcultures of two different isolates. Such isolates were regarded as indistinguishable in PMS. The principles of the analysis have been described previously. ${ }^{9}$

Representative isolates (one from each patient) of the clusters identified in Batches 1, 2 and 3 were repyrolysed in a single machine run. These data were analysed as described above in order to assess whether the strains representing each cluster were distinct.
Finally, 18 isolates, comprising three from the cluster identified in Batch 1, seven from the cluster in Batch 2, and four from the cluster in Batch 3 (i.e., all from the north west of England) were again reanalysed along with four isolates representing a cluster in Batch 4 (from the north east of England) to investigate similarities and differences between the putative outbreak strains in the geographically separate regions. These data were used in a UPGMA analysis to construct a dendrogram based on Mahalonobis distances.

\section{Results}

Fig. 1a is the ordination diagram obtained from Batch 1, containing the isolates from the suspected outbreak in the GAU. Isolates GAU 4, 5 and 10 appeared to be distinguishable; the remainder formed a tight cluster including the two isolates from the same patient (GAU 7a and 7b). In the ordination diagram from Batch 2 (fig 1b; LSG isolates) isolates LSG 9 and 12 clearly differed from the remainder, which formed a tight cluster, including the repeat isolates of the LSG 2, LSG 5, LSG 8, LSG 11, LSG 13, LSG 15 and LSG 16 sets. Analysis of Batch 3 (not shown) showed that isolates LSG 90/1, LSG 90/2, LSG 90/4 and GM $90 / 2$ formed a tight cluster; other isolates were distinguishable from the cluster and from each other.

Fig. $1 \mathrm{c}$ is the ordination diagram resulting from the re-examination of isolates representing the clusters from Batches 1, 2 and 3. Although there were three groups on the ordination diagram broadly corresponding to the three sources, seven of the isolates from the LSG cluster were located with the GAU isolates. By contrast, the five isolates from 1990,

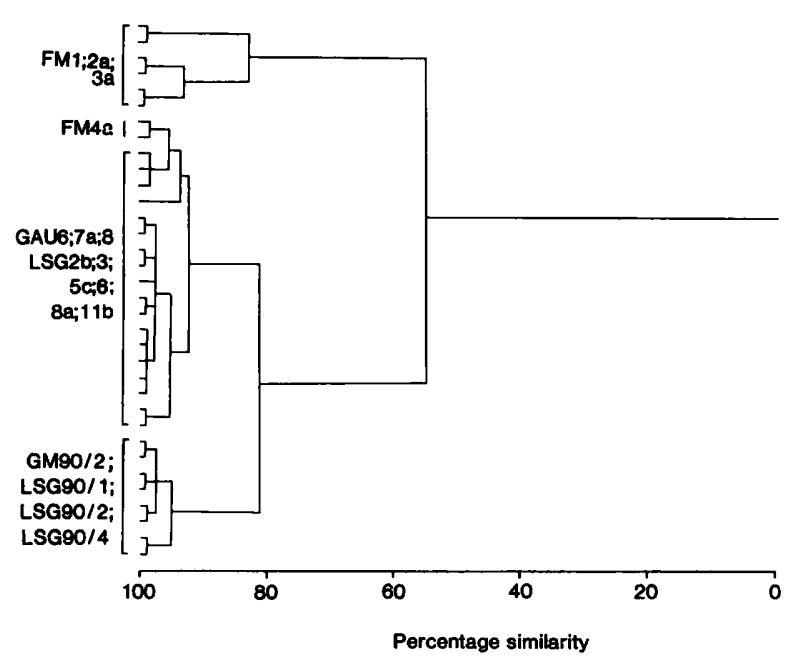

Fig. 2. A dendrogram derived from PMS data showing percentage similarity of representative isolates from outbreaks of $C$. difficileassociated diarrhoea and colitis in (a) the north east of England (isolates FM 1, 2a, 3a and 4a), (b) the north west of England in 1991 (isolates GAU 6, 7a, 8 and LSG 2b, 3, 5c, 6, 8a and 11 ), and (c) the north west of England in 1990 (isolates GM 90/2 and LSG 90/1, $90 / 2$ and $90 / 4)$. The clustering method was UPGMA. 
including four isolates from the LSG hospital, were more similar to each other than the other groups. Nonetheless, the Mahalanobis distances indicated that the differences between duplicate subcultures of the same isolate were often as large as those between subcultures of different isolates. All 26 isolates were, therefore, very closely similar.

Fig. 1d is the ordination diagram from the analysis of Batch 4, comprising the isolates from outbreak 2. Isolates HAEM 2 and ORTH were markedly different from the remaining isolates that formed a tight cluster. This cluster contained all isolates and repeat isolates from the affected patients on the female medical ward, but also included one isolate from an apparently epidemiologically unrelated source (HAEM 1).

Representative isolates from different years in the north west (NW), and also NW isolates with north east (NE) isolates, are compared in the dendrogram (fig. 2). The NE isolates were, with one exception, quite distinct from the NW isolates. The close similarity between isolates from the GAU and LSG in 1991 was confirmed and the previous suggestion (fig. 1d), that LSG isolates in 1990 may be distinguishable from those in 1991, was strengthened.

\section{Discussion}

These results show that PMS can be used to investigate suspected outbreaks of infection with $C$. difficile to determine if cross-infection with a single strain is occurring. In outbreak 1 the first analysis of the GAU isolates showed that eight of 11 isolates were indistinguishable in PMS, including a repeat isolate from a patient who had remained symptomatic for over a month. Analysis of the 24 isolates from the long stay geriatric hospital to which GAU patients were sent, and in which an increase in C. difficile-associated disease was noted shortly after the suspected outbreak in the acute hospital GAU, showed that 22 of these isolates were indistinguishable, again including several repeat isolates from patients with persistent disease.

When representatives of these two clusters of related isolates were compared, analysis suggested that all the isolates were closely similar and, therefore, that a single strain of $C$. difficile was likely to have been involved at both sites. The results strongly supported epidemiological evidence that an outbreak had arisen in the GAU, closely followed by a consequential outbreak in the LSG hospital.

PMS was also able to discern a cluster of closely related isolates from an outbreak on a female medical ward in a different hospital (outbreak 2) re-emphasising the ability of the technique to confirm rapidly the presence of an outbreak. The inclusion within this cluster of an apparently epidemiologically unrelated isolate may simply be a coincidental sporadic case due to the same strain as that causing the outbreak elsewhere in the same institution.

The overall similarity but discernible differences between isolates from the LSG hospital in 1990 and isolates representing the outbreak strain from the same site in 1991 may represent small changes in a single strain during passage through many patients over several months, implying that the increased incidence in symptomatic disease in Feb.-March 1991 was due to host-related factors or changes in patient care facilitating spread of the organism. Alternatively, the small but discernible differences between the LSG 1990 isolates and those from 1991 might be associated with a change in the virulence of the strain. Strainspecific virulence factors in $C$. difficile have been postulated, ${ }^{10}$ although not yet substantiated. ${ }^{11}$ PMS also demonstrated considerable differences between representative isolates from the two outbreak strains in two widely separate geographical locations in the North of England in different years.

The increasing recognition that nosocomial spread of $C$. difficile occurs makes it important that apparent increases in the incidence of $C$. difficile-associated disease are rapidly investigated for evidence of crossinfection. The introduction of enteric infection control measures may limit spread and prevent further cases. ${ }^{12}$ C. difficile can currently be typed by various methods, including SDS-PAGE autoradiography, ${ }^{2}$ immunoblotting, ${ }^{3}$ serotyping, ${ }^{4}$ phage typing ${ }^{5}$ and restrictionendonuclease analysis (REA). ${ }^{6}$ Some of these methods have limitations in sensitivity and specificity and the two most discriminatory techniques (autoradiography and REA) are relatively expensive.

In this context another rapid and discriminatory method of inter-strain comparison applicable to $C$. difficile may be of considerable use. PMS is rapid and capable of handling large numbers of organisms in a single analysis. Up to 50 isolates can be examined within $24 \mathrm{~h}$, each in duplicate subculture and each subculture in triplicate. Although initial capital costs are high, consumable costs are low and the same apparatus can be used without adaptation for a wide range of bacterial species. The major disadvantage of PMS is that it is currently unable to assign permanent type designations. Nonetheless, PMS, where available, may be a good initial screening method for suspected nosocomial infection with $C$. difficile. The use of such a rapid, high volume method could complement other techniques, allowing more expensive methods to be focused to maximum effect, whilst also providing the immediate information necessary for outbreak management.

We thank Dr V. Khanna, Consultant Geriatrician, North Manchester General Hospital and Drs S. J. Pedler and A. J. Bint, Consultant Microbiologists, Royal Victoria Infirmary, Newcastle upon Tyne, for their help. 


\section{References}

1. Bender BS, Bennett $\mathrm{R}$, Laughon $\mathrm{BE}$ et al. Is Clostridium difficile endemic in chronic-care facilities? Lancet 1986; 2: 11-13.

2. Tabaqchali S, Holland D, O'Farrell S, Silman R. Typing scheme for Clostridium difficile: its application in clinical and epidemiological studies. Lancet 1984; 1: 935-938.

3. Mulligan ME, Peterson LR, Kwok RYY, Clabots CR, Gerding DN. Immunoblots and plasmid fingerprints compared with serotyping and polyacrylamide gel electrophoresis for typing Clostridium difficile. J Clin Microbiol 1988; 26: 41-46.

4. Toma S, Lesiak G, Magus M, Ho-Lau L, Delmée M. Serotyping of Clostridium difficile. J Clin Microbiol 1988; 26: 426-428.

5. Sell TL, Schaberg DR, Fekety FR. Bacteriophage and bacteriocin typing scheme for Clostridium difficile. J Clin Microbiol 1983; 17: 1148-1152.

6. Kuijper EJ, Oudbier JH, Stuifbergen WNHM, Jansz A, Zanen HC. Application of whole-cell DNA restriction endonuclease profiles to the epidemiology of Clostridium difficile-induced diarrhea. J Clin Microbiol 1987; 25: 751-753.

7. Wren BW, Tabaqchali S. Restriction endonuclease DNA analysis of Clostridium difficile. J Clin Microbiol 1987; 25: 2402-2404.

8. Freeman R, Goodfellow M, Gould FK, Hudson SJ, Lightfoot NF. Pyrolysis-mass spectrometry (Py-MS) for the rapid epidemiological typing of clinically significant bacterial pathogens. J Med Microbiol 1990; 32: 283-286.

9. Orr K, Gould FK, Sisson PR, Lightfoot NF, Freeman R, Burdess D. Rapid inter-strain comparison by pyrolysis mass spectrometry in nosocomial infection with Xanthomonas maltophilia. $J$ Hosp Infect 1991; 17: 187-195.

10. McKay I, Coia JE, Poxton IR. Typing of Clostridium difficile causing diarrhoea in an orthopaedic ward. J Clin Pathol 1989 ; 42 : 511-515.

11. McFarland LV, Elmer GW, Stamm WE, Mulligan ME. Correlation of immunoblot type, enterotoxin production, and cytotoxin production with clinical manifestations of Clostridium difficile infection in a cohort of hospitalized patients. Infect Immun 1991 ; 59: 2456-2462.

12. Testore GP, Pantosti A, Cerquetti M, Babudieri S, Panichi G, Gianfrilli PM. Evidence for cross-infection in an outbreak of Clostridium difficile-associated diarrhoea in a surgical unit. J Med Microbiol 1988; 26: 125-128. 